\title{
Differences of opinion on the effectiveness of working a 12 step program a survey study
}

\author{
Abstract \\ Background: In the above quotation, Dr. William \& D. Silkworth suggested that a \\ spiritual cure for alcohol addiction could be achieved by twelve steps of recovery. \\ Today, Alcoholics Anonymous (AA) exists in approximately 170 countries, carrying \\ its message to over 114,000 groups and millions of people.
}

Purpose: To study the perceived subjective and objective effect of the AA program on participants. Method: An anonymous survey of clinicians and substance abuse sufferers on the effects of the AA program.

Results: Patients are more enthusiastic about the benefits of the program than are psychiatrists.

Conclusion: People who struggle with substance use disorders find more benefit in AA services than clinicians realize.

"Once a psychic change has occurred, the very same person who seemed doomed, who had so many problems he despaired of ever solving them, suddenly finds himself easily able to control his desire for alcohol, the only effort necessary being that required to follow a few simple rules."

\section{--William D. Silkworth}

Keywords:addiction, alcoholics, substance abuse, psychiatrists, enthusiastic, symptoms
Volume 3 Issue 4 - 2017

\author{
Paulina Riess, Panagiota Korenis
}

Department of Psychiatry, USA

Correspondence: Paulina Riess, Department of Psychiatry, Bronx Lebanon Hospital Center, 1276 Fulton Ave Bronx, NY 10456, USA, Tel 917-698-0653, Email priess@bronxleb.org

Received: May 09, 2017| Published: May 25, 2017

\section{Introduction}

The fellowship of Alcoholics Anonymous was founded in 1935. Bill Wilson and Dr. Bob Smith developed the 12 steps as a program of spirituality and character development to treat what is now known as alcohol use disorder. ${ }^{1}$ As of 2016 , the fellowship has over two million members. In a letter to Bill Wilson, the famous psychoanalyst Carl Jung described two ways in which individuals suffering from addiction might recover. One way included religious insight, and the other consisted of personal, honest contact with friends. ${ }^{2}$ Evidence suggests that use of the substance of choice decreases in the context of working a 12 step program. Authors of one study point out that self-reported craving was inversely related to involvement in AA. ${ }^{3}$ Authors of a study that employed a large clinical sample of individuals with alcohol use disorder concluded that working the AA 12 step program leads to improvements in alcohol use as well as psychological and emotional well-being. ${ }^{4}$ This notion is based upon the emotional dysregulation, depression, and anxiety that often accompany active addition. Dr. Silkworth selected the terms "restless, irritable, and discontent" to describe mood symptoms often associated with addiction. He emphasized not only a recovery from consumption of alcohol but from feelings of hopelessness. ${ }^{1}$ Authors of the study previously mentioned have found that individuals who actively worked a 12 step program experienced a reduction in depressive symptoms. ${ }^{4}$ Bergman and his group conclude that involvement in a 12 step program may help offset potentially worse substance use outcomes related to comorbid psychiatric illness. ${ }^{5}$ This point is once again emphasized in yet another study in which the authors state that affiliation with Alcoholics Anonymous predicted better proximal treatment outcomes including sustained self-efficacy, motivation to refrain from use, and increased active coping efforts. ${ }^{6}$
Alcoholics Anonymous is not opposed to medical treatment of substance use disorders. In fact, according to its $10^{\text {th }}$ tradition, Alcoholics Anonymous has no opinion on outside issues. Concurrent treatment of comorbid psychiatric illness as well as medical treatment of addiction itself is encouraged in AA literature. ${ }^{1}$ Dr. Silkworth elaborated on the need for hospital procedure and psychological measure in the treatment of substance use disorder. The effectiveness of medical management of addiction varies according to published literature. Howland et al. conclude that efficacy of medication has typically been established on short term studies, but information about long term adherence, efficacy, and safety is lacking. ${ }^{7}$ AA literature emphasizes the need for a willingness to recover, without which the program does not work. ${ }^{1}$ Because AA members and clinicians often view the benefit of a service differently, we set out to survey clinicians' and substance abuse sufferers' opinions as to the gains they perceived from the AA program. ${ }^{8-10}$

\section{Methods}

After obtaining IRB approval from Bronx Lebanon Hospital Center in the Bronx, New York, fifty anonymous surveys consisting of 22 questions were distributed among individuals attending AA meetings in the community, as well as individuals on an inpatient psychiatric unit diagnosed with substance use disorders. To prepare the survey, a literature search was conducted in order to determine if an evidence-based tool existed that assessed satisfaction with services of clinicians and people with substance use disorders.

After doing a literature search in various search engines including PubMed, GoogleScholar and PsycInfo no tools specific to our enquiry were found. Questions were then developed using the current literature 
related to twelve step programs. The clinicians (psychiatrists and psychiatric residents) worked directly with patients with substance use disorders.

\section{Patient samples were determined in two ways}

a. Patients who were admitted to the Inpatient Service who were diagnosed with a Substance Use Diagnosis using DSM-IVTR and DSM 5 criteria.

b. People who participated in a Twelve Step program in the community. Individuals were randomly approached by IRB approved personnel and asked to participate in the survey. Of the 50 surveys distributed, 44 individuals agreed to participate and 6 refused. Questions were written in multiple choice formats with space available for elaboration in writing.

Fifty anonymous surveys consisting of 25 questions were distributed among psychiatrists of various subspecialties and levels of training at a New York hospital. Of the 50 surveys distributed, 42 individuals chose to participate. Questions varied from level of training, subspecialty if applicable, mood symptoms in substance use disorder patients, knowledge of 12 step programs including Alcoholics Anonymous, as well as likelihood of recommending participation in a 12 step program to substance use disorder patients. Questions were written in multiple choice formats with space available for elaboration in writing.

\section{Qualitative results}

\section{Participant non-clinicians}

The sample was 26 male; 18 female; predominantly white; the majority with less than five years of sobriety; 39 AA members with 36 having worked the 12 steps with a sponsor. 40 experienced mood symptoms with 30 having felt suicidal with or without prior attempted suicide in the past.

\section{Mood}

Thirty-one non-clinician participants attributed their mood symptoms to alcoholism. Thirty-six felt that their mood symptoms decreased after working a 12 step program. Of the 44 participants, $81 \%$ had seen a psychiatrist with $68 \%$ receiving a diagnosis of a Mood Disorder. $77 \%$ were treated with psychotropic medication. Of those that were prescribed medication, $78 \%$ felt that working a twelve step program with a sponsor and attending meetings on a regular basis was most effective in reducing their mood symptoms. $25 \%$ of individuals felt that the combination of both medication and the twelve step program was effective in reducing their mood symptoms.

Participants elaborated on their answers as follows: One person felt that once he/she began working a twelve step program, they no longer required psychotropic medication. 5 individuals felt that they have been misdiagnosed with a mood disorder and treated with medication with ineffective results.

\section{Relationship with psychiatrist}

Of the 44 participants, 10 felt that their present or past psychiatrists treated their substance use disorder as secondary to another mental illness. 10 individuals felt that they have been misdiagnosed by a psychiatrist in the past. 13 individuals felt that their inpatient/outpatient psychiatrists placed no emphasis on the importance of attending 12 step meetings, 6 answered minimal emphasis, 20 answered a lot of emphasis. 3 individuals felt that their past/present psychiatrists knew nothing about their substance use disorder. 20 individuals felt that their psychiatrists knew a little about their substance use disorder. 14 individuals felt that their psychiatrists knew a lot about their substance use disorder. 2 individuals felt that their psychiatrists had no knowledge of 12 step programs. 17 individuals felt that their psychiatrists had little knowledge of 12 step programs. 16 individuals felt that their psychiatrists were well versed regarding 12 step programs.

\section{Participant clinicians}

Opinions about 12 step programs: Of the 42 psychiatrists who participated in completing the survey: 16 identified as Attending, 4 identified as Fellows, and 22 identified as Residents. Subspecialties included the following: Addiction Psychiatry: 1, Forensic Psychiatry: 2, Child Psychiatry: 5, Consultation Liaison Psychiatry: 6, Geriatric Psychiatry: 1. 41 individuals felt that Substance Use is a disease. 30 individuals felt that substance use disorders are not related to willpower. 10 individuals felt that substance use disorders are related to willpower. 13 individuals answered that there is no verbatim definition of "an Alcoholic" according to 12 step literature, 24 individuals were not familiar with this concept. 28 psychiatrists felt that most of their substance use disorder patients experience mood symptoms. Only 8 psychiatrists stated that they would recommend working a 12 step program as a primary form of treating mood symptoms in substance use disorder patients. 25 psychiatrists felt that they have misdiagnosed a substance use disorder patient in the past. 27 psychiatrists felt that medication they have prescribed to substance use disorder patients may not have been conducive to their sobriety.

Familiarity with 12 step programs: 12 psychiatrists felt that they were very familiar with the concepts of Alcoholics Anonymous and other 12 step programs. 27 psychiatrists felt that AA and other 12 step programs are crucial in achieving and maintaining sobriety. 1 individual felt that AA and other 12 step programs are not important at all in achieving and maintaining sobriety. 13 individuals felt that AA and other 12 step programs are somewhat important in achieving and maintaining sobriety. 19 individuals answered that they always recommend getting a sponsor and going to meetings to substance use disorder patients, 22 individuals sometimes recommend 12 steps participation, and 1 person never recommended this method.

Of the 42 psychiatrists who participated in completing the survey, 24 were familiar with the concept that Alcoholics Anonymous and other 12 step programs are programs of attraction rather than promotion and do not recruit members. 18 psychiatrists were familiar with the Big Book as the primary text of AA, and 28 correctly identified AA's founders as Bill Wilson and Dr. Bob Smith. 40 individuals were familiar with the concept of sponsorship. 19 out of 42 individuals were familiar with the concept that working a 12 step program is not finite and attending meetings on a daily basis over a lifetime is strongly suggested in AA literature.

\section{Discussion}

A majority of the individuals who participated in our survey endorsed the fact that working a 12 step program was effective in the achievement of sobriety and also in treating associated mood symptoms. Participants described improvements in symptoms related to depression and anxiety as well as decreased impulsivity and cravings. These individuals were actively involved in AA and worked closely with a sponsor. A majority of the psychiatrists who participated 
in our study felt that addiction is a disease and is not, therefore, related to willpower. However, approximately half stated that involvement in a 12 step program is important in the treatment of addiction. Only half of the physician participants encourage their patients to attend AA meetings. Physician knowledge pertaining to the program was limited. Based on our findings, we feel that psychiatrists would benefit from education regarding the importance of encouraging their patients to become actively involved in AA and other 12 step programs. Our findings suggest that individuals with substance use disorders find working a 12 step program beneficial and that not enough psychiatrists understand and emphasize this in their management.

\section{Acknowledgements}

None.

\section{Conflict of interest}

The author declares no conflict of interest.

\section{References}

1. Alcoholics Anonymous. 4th ed. New York: AA World Services; 2001.

2. Kelly JF, Stout RL, Magill M, et al. Mechanisms of Behavior Change in Alcoholics Anonymous: Does AA lead to better alcohol use outcomes by reducing depression symptoms? Addiction. 2010;105(4):626-636.
3. Galanter M, Josipovic Z, Dermatis H, et al. An Initial fMRI Study on Neural Correlates of Prayer in Members of Alcoholics Anonymous. Am J Drug Alcohol Abuse. 2017;43(1):44-54.

4. Kelly JF, Hoeppner B, Stout RL, et al. Determining the Relative Importance of the Mechanisms of Behavior Change within Alcoholics Anonymous: A Multiple Mediator Analysis. Addiction. 2011;107(2):289-299.

5. Bergman GB, Greene MC, Hoeppner BB, et al. Psychiatric Comorbidity And 12-Step Participation: A Longitudinal Investigation Of Treated Young Adults. Alcohol Clin Exp Res. 2014;38(2):501-510.

6. Morgenstern J, Labouvie E, McCrady BS, et al. Affiliation with Alcoholics Anonymous after treatment: a study of its therapeutic effects and mechanisms of action. J Consult Clin Psychol. 1997;65(5):768-777.

7. Howland RH. Evidence and the Treatment of alcohol use disorder. $J$ Psychosoc Nurs Ment Health Serv. 2015;53(5):11-14.

8. Stevens EB, Jason LA. Evaluating alcoholics anonymous sponsor attributes using conjoint analysis. Addict Behav. 2015;51:12-17.

9. Wilcox CE, Tonigan JS. Changes in depression mediate the effects of AA attendance on alcohol use outcomes. Am J Drug Alcohol Abuse. 2016;28:1-10.

10. Jellinek EM. Phases in the Drinking History of Alcoholics. $Q J$ Stud Alcohol. 1946;7:1-88. 